\title{
ARCHIVOS ESCOLARES QUE CONSTRUYEN IDENTIDAD: EL LICEO PÚBLICO, HISTORIA Y PATRIMONIO DE LOS CHILENOS
}

\author{
Rodrigo Esteban Sandoval Díaz \\ Pontificia Universidad Católica de Chile \\ rsandovd@uc.cl \\ María José Vial \\ Pontificia Universidad Católica de Chile mvially@uc.cl
}

\begin{abstract}
RESUMEN
Históricamente se ha asignado al liceo público chileno el rol de ser un espacio de aprendizaje y formación ciudadana. Si bien, desde su fundación en 1813 fue el lugar por excelencia de formación para los futuros dirigentes de la nueva república, el aumento de la cobertura y el creciente acceso de otros sectores de la sociedad transformaron el tipo de estudiantes matriculados en sus aulas, aunque no su misión original. Hoy, en pleno desarrollo de la sociedad del conocimiento, el desafío de formar sociedades integradas por personas informadas, participativas y respetuosas sigue siendo asunto prioritario de esta y de cualquier institución escolar. El presente proyecto, basado en el rescate y difusión de los archivos documentales conservados por los liceos públicos del país, nace de la necesidad de generar instancias colaborativas de investigación, participación y construcción de memoria histórica para estudiantes y profesores de educación secundaria.
\end{abstract}

Palabras clave: Archivos escolares. Ciudadanía. Educación. Memoria histórica. Identidad.

\section{FILES SCHOOL BUILDING IDENTITY: THE PUBLIC HIGH SCHOOL HISTORY AND HERITAGE OF CHILEAN}

\begin{abstract}
Historically, the role of learning and civic formation has been asigned to the chilean public lyceum. Since its foundation in 1813, it became the place chosen by excellence for the education of the future leaders of the new Republic. However, the increase in its coverage and the growing access of other sectors of the society transformed the kind of students registered in its classrooms, although not its original mission. Today, in the midst of the knowledge society, the challenge to form communities integrated by informed, participating and respectfull persons is still a priority of this and any other teaching institution. This proyect, based in the rescue and dissemination of the documentary archives kept in the public lyceums of the country, emerges from the need to create cooperative instances for research, participation and historic memory construction for students and teachers of secondary education.
\end{abstract}

Keywords: School records; citizenship; education; historic memory; identity. 


\title{
ARQUIVOS ESCOLARES QUE CONTROEM IDENTIDADE: LICEU PÚBLICO, HISTÓRIA E PATRIMÔNIO DOS CHILENOS
}

\begin{abstract}
RESUMO
Historicamente tem sido atribuído à escola pública chilena o papel de ser um espaço de aprendizagem e formação para a cidadania. Embora desde a sua fundação em 1813 fosse um lugar por excelência de formação para os futuros líderes da nova república, o aumento da cobertura e do crescente acesso a outros setores da sociedade transformaram o tipo de alunos matriculados em suas salas de aula, ainda que não fosse sua missão original. Hoje, em pleno desenvolvimento da sociedade do conhecimento, o desafio de formar sociedades integradas por pessoas informadas, participativas e respeitosas continua a ser um assunto prioritário nesta e em qualquer instituição de ensino. O presente projeto, com base no resgate e difusão dos arquivos documentais conservados por liceus públicos do país, nasce da necessidade de gerar instâncias colaborativas de investigação, participação e construção da memória histórica para estudantes e professores do ensino secundário.
\end{abstract}

Palavras-chave: Arquivos escolares. Cidadania. Educação. Memória histórica. Identidade.

\section{INTRODUCCIÓN}

Históricamente se ha asignado al liceo público el rol de ser un espacio de aprendizaje y formación ciudadana. A partir de la fundación del Instituto Nacional en 1813, el liceo fiscal masculino chileno se configuró como la principal institución educativa secundaria del país. El primer establecimiento republicano, civil y eclesiástico, que fundió a las instituciones educativas del periodo colonial pretendía "crear una generación virtuosa y social por principios [ ], reunir los conocimientos y las luces en un centro común para que se propaguen uniformes [donde los jóvenes] afianzarán su fe, fortalecerán su espíritu y conocerán sus obligaciones"1. Con los años, el plantel se convirtió en el modelo para todos los liceos creados durante el siglo XIX en las ciudades principales de las provincias chilenas.

El liceo público fue desde un comienzo centralizado, uniforme, urbano, elitista y conducente a la educación superior. Su acción reforzó al Estado docente, fue espacio propicio para la difusión de la nacionalidad e instrumento activo de integración territorial y social. Territorial, porque si bien la tónica de propagación del liceo se concentró en los centros urbanos

\footnotetext{
${ }^{1}$ Proclama del Senado conservador sobre el restablecimiento del Instituto en 1819. En Centenario del Instituto Nacional, 1813-1913. Breve reseña histórica redactada por encargo del señor rector Juan N. Espejo. Imprenta, Litografía y Encuadernación Barcelona, Santiago de Chile, 1913.
} 
de mayor tamaño de la zona central, a partir de la década de 1860 se expandió hacia lugares donde debía cumplir un rol de soberanía y chilenización en zonas en proceso de ocupaciónAraucanía- o de reciente anexión al país -Tarapacá y Antofagasta-. Y social, ya que devino en un espacio de confluencia relativamente heterogéneo, permitiendo a nuevos grupos de la sociedad acceder al conocimiento y a niveles superiores de educación. Junto con la escuela primaria, configuró la primera red del sistema de educación pública que desempeñó un papel fundamental en la conformación de identidades locales ya que una parte significativa de la población nacional pasó por sus aulas.

El liceo visto como "un lugar de memoria escolar y pesquisa histórica" (MENEZES; SILVA; TEIXEIRA JÚNIOR, 2005) mediante el reconocimiento y la investigación en torno a su herencia documental es uno de los propósitos de este proyecto. Como toda institución, este generó un cuerpo de documentos desde el momento de su fundación, fuentes que por sus contenidos permiten reconstruir vivencias y representaciones de la experiencia escolar además de la vida cotidiana, cultural y administrativa "posibilitando las lecturas y relecturas del pasado con vistas a las necesidades y objetivos del presente" (LUZ, 2014). Son los libros de gestión escolar que la legislación vigente en cada época demandaba llevar, y que suministran hoy una radiografía institucional y social del sistema de educación público chileno desde sus inicios. Actas de exámenes y libros de matrícula de alumnos externos, internos o "medio pupilo" registran fecha de incorporación, nombre de padres o apoderados, lugar de nacimiento, edad, domicilio, y clases que cursa. Libros de vida y fichas individuales de los estudiantes que consignaban los datos del alumnado con sus calificaciones y observaciones en forma semanal. Copiador de oficios y correspondencia enviada y recibida; actas de las sesiones del Consejo de Profesores, donde se discutía sobre alumnos aventajados y asuntos pedagógicos tales como textos de estudio, programas y régimen interno. A esto, se suman materias particulares como actas de asociaciones estudiantiles, registros de liceos nocturnos, información sobre los internados, hojas de servicio de profesores. El archivo documental permite recrear un panorama en el que avistamos a profesores, estudiantes y administrativos, la vida cotidiana dentro de los establecimientos y del aula; la cultura material de la educación en su real dimensión; la sociología de los estudiantes y sus familias; las prácticas pedagógicas y características de la relación pedagógica; la asociatividad estudiantil y la formación de la cultura política; la formalización de instituciones intermedias 
como centros de padres y centros de alumnos. Finalmente, los vínculos entre las distintas autoridades en el proceso de construcción del Estado Docente.

En los últimos años, diversas instituciones han desplegado planteamientos y acciones en pos de la recuperación de su patrimonio documental (archivos y bibliotecas históricas) y mueble (objetos pedagógicos y mobiliario). Estas iniciativas han posibilitado el inicio de la indagación histórica, cultural y patrimonial en los establecimientos que las dirigen y permiten identificar en los corpus patrimoniales tanto recursos para la historia como elementos para la memoria e identidad de las comunidades. Entre 2004 y 2007 el Consejo de Monumentos Nacionales invitó a los establecimientos educacionales a declarar sus patrimonios muebles e inmuebles como Monumento Histórico ${ }^{2}$. Si bien se privilegió la declaratoria de bienes inmuebles, se sumaron también bienes muebles de algunas instituciones escolares secundarias en su mayoría de la capital del país. Mobiliario, pinturas, libros, instrumentos de ciencias, entre otros, cubrieron la mayor parte de la declaratoria. El pronunciamiento sobre documentos de archivo fue extremadamente escaso. El mayor corpus documental declarado lo posee el ya citado Instituto Nacional General José Miguel Carrera de Santiago y consta de libros de matrícula, certificados y correspondencia entre los años 1813 y 1899, además de una serie de fotografías ${ }^{3}$. Para regiones sin embargo, solo existe una declaratoria de bienes muebles, la del Liceo Polivalente Sara Braun de Punta Arenas, que contiene documentos de Gabriela Mistral, una colección de fotografía y cuatro aparadores de libros. Ciertamente, la presencia de documentos producidos por planteles educativos es insignificante en el ámbito institucional estatal chileno de declaración patrimonial.

El archivo escolar, definido como "la institución cultural donde se reúne, conserva, ordena y difunden los conjuntos orgánicos de documentos para la gestión administrativa, la información, la investigación y la cultura”, no existe en la mayor parte de los establecimientos educacionales públicos del país. Realizar un aporte al desarrollo de la cultura local abordando el estudio sobre la situación de estos archivos es nuestro desafío. Su recuperación implica develar un dossier de fuentes históricas inéditas para la renovación de la historia de la educación, la

\footnotetext{
${ }^{2}$ Realizado por el Programa de Patrimonio Educativo del Ministerio de Educación de Chile desarrollado entre 2004 y 2007 a través del Consejo de Monumentos Nacionales, CMN, y UNESCO,

${ }_{3}$ Decreto Exento 337/2004, Ministerio de Educación de Chile, Declara Monumento Histórico las colecciones de libros, óleos y bienes muebles que indica pertenecientes al Instituto Nacional José Miguel Carrera. En <http://www.monumentos.cl>, consulta 01 mar. 2016. El Programa de Archivos Escolares junto a la Academia de Ciencias Sociales del IN elaboró un inventario general que completa la información disponible en la declaratoria.
} 
innovación en la enseñanza de la Historia y, lo más relevante, para el trabajo participativo desde las comunidades escolares en torno a su memoria e identidad. Junto con plantear estrategias para la conservación de los depósitos documentales, se busca entregar herramientas para activar la consolidación de una Red Nacional de Archivos Escolares en Chile. La primera etapa considera únicamente los archivos de los liceos fundados entre 1813 y 1909, pero la iniciativa pretende ampliar su espectro temporal y abordar los fondos de escuelas primarias o básicas.

La reconstrucción del legado y del significado del liceo público chileno a través de sus fuentes, y en conjunto con estudiantes, profesores e investigadores, implica la revisión de una pieza clave dentro de la historia regional y nacional del país, plena de elementos de referencias culturales y de pertenencia. Si bien la historiografía y el trabajo con el patrimonio material han contribuido al desarrollo de la cultura local, innovar en su estudio mediante la puesta en valor, utilización y difusión de los archivos de los liceos con y para alumnos y docentes contribuye a la reconstrucción de la memoria que define vínculos de identidad, en este caso entre la comunidad y la institución escolar. A través del encuentro activo e inclusivo entre la ciudadanía, su historia y su memoria es posible enriquecer la comprensión histórica entre un público externo a la academia (LEON, 2010). El foco es incidir en la formación ciudadana que implica, precisamente, el conocimiento y la valoración por parte de los jóvenes de las instituciones públicas y privadas, desde las más cercanas, como la escuela, y vinculadas a su vida cotidiana, como un piso esencial para promover el respeto y valoración de las instituciones del Estado, la vida en democracia y la convivencia social. A su vez, incentiva el trabajo de la comunidad escolar en su conjunto en pos del propio patrimonio y promueve la discusión y desarrollo de políticas públicas vinculadas al ámbito de los archivos, el patrimonio y la educación.

Las tradicionales visiones del patrimonio hacen hincapié en las expresiones objetuales y menos en comprometer a las comunidades escolares en la reflexión sobre el porqué del patrimonio y la necesaria reflexión sobre sí mismas. El ejercicio desarrollado en diversos liceos ha permitido el diálogo y cooperación entre personas de diversos campos disciplinares tales como historiadores, archiveros, profesores, gestores culturales y la comunidad escolar, que ha comprendido el interés y el significado profundo de su acervo documental como posibilidad para crear y difundir un material de interés pedagógico e investigativo innovador dada la densidad histórica de su contenido y el valor educativo, cultural y social que encierra. 


\section{UNA RUTA INNOVADORA: PROGRAMA DE ARCHIVOS ESCOLARES, PAE}

El 27 de febrero de 2010 gran parte de Chile amaneció en medio de la devastación. Un terremoto de magnitud inusitada destruyó vidas, proyectos y gran parte de la infraestructura del país. La red educativa pública de la zona centro sur fue uno de los sectores más afectados y debió evacuar la mayoría de sus planteles debido a los daños ocasionados en sus espacios y edificios. El proceso de recuperación fue lento y complejo, y las comunidades escolares debieron funcionar por un largo tiempo en lugares provisionales mientras el Estado y la sociedad luchaban por salir del caos que esto significaba para el medio educativo. La situación de extremo riesgo del patrimonio en su sentido más amplio, movilizó a un equipo de investigadores y estudiantes del Instituto de Historia de la Universidad Católica de Chile que al poco andar decidió crear el Programa de Archivos Escolares para recuperar la documentación histórica de los liceos. Su primera intervención se desarrolló en el Liceo Abate Molina, el primero de hombres fundado en la ciudad de Talca, ubicada a unos trecientos kilómetros de la capital. Durante dos años, investigadores junto a los alumnos inscritos en el curso de Historia y Archivos $^{4}$ viajaron a Talca para recuperar y organizar el valioso archivo conservado en las antiguas dependencias del liceo. El trabajo se realizó en conjunto con profesores y alumnos del propio colegio que en junio de 2012, en medio de una ceremonia pública llena de alusiones a la relevancia del rol cumplido por el centenario plantel, y mientras las clases continuaban dictándose dentro de containers de fierro provisionales instalados en el patio central del edificio, inauguraron el Archivo LAM. Tras las huellas de nuestra identidad docente y estudiantil: Archivo del Liceo Abate Molina de Talca, una pieza clave en la historia escolar de Chile, se llamó ese primer proyecto colaborativo de un equipo que hoy está comprometido no solo con la recuperación de este acervo a nivel nacional sino con la contribución que ello significa para la formación de los jóvenes ciudadanos ${ }^{5}$.

¿Qué es un archivo escolar? ¿Existen otros archivos de este tipo en Chile? ¿Conservan las instituciones educativas sus documentos como un patrimonio que incumbe no solo a la

\footnotetext{
${ }^{4}$ Historia y Archivos es un curso optativo de profundización que imparte el Instituto de Historia de la Pontificia Universidad Católica de Chile. Desarrolla conceptos básicos sobre el tratamiento de los documentos de archivo, valoración del patrimonio documental y análisis de la situación de los archivos y la archivística en Chile.

${ }^{5}$ El Archivo LAM, ubicado junto a la biblioteca del liceo contó con el financiamiento del Programa para Bibliotecas y Archivos Latinoamericanos de la Universidad de Harvard. <www.archivosescolares.cl>, <www.lam.cl>.
} 
historia y a la cultura local sino a toda la sociedad? ¿Es necesario preservar los documentos generados por los establecimientos educacionales?

Estas fueron algunas de las preguntas que dieron vida al Programa de Archivos Escolares cuyo objetivo es levantar una red de liceos emblemáticos comprometidos con la conservación y difusión de su patrimonio documental a través del censo, recuperación y organización de sus archivos y colecciones históricas. Una vez creados los espacios y las condiciones para el desarrollo de un trabajo colaborativo dentro de los liceos de la red, se busca forjar procesos de aprendizaje y conocimiento, apropiación y valorización de esta herencia cultural inexplorada con la participación de estudiantes, profesores e investigadores.

El programa surgió desde un equipo multidisciplinario liderado por historiadores que investiga nuevas perspectivas y un conocimiento actualizado sobre la historia de la educación en Chile (SERRANO et al., 2012). Fue desarrollado como una línea de extensión del proyecto "La educación ante el riesgo de fragmentación social: ciudadanía, equidad e identidad nacional" 2008 al 2012- en pos de generar instancias de vinculación entre la academia y el aula al producir conocimiento histórico sobre la educación y canalizarlo a través de distintos recursos pedagógicos hacia el profesorado, impactando en la revalorización de la labor docente, específicamente en el área de Ciencias Sociales e Historia. El descubrimiento de la existencia de fondos documentales escolares públicos inéditos, muchos de ellos regionales, dio pie a la creación del programa por las posibilidades de levantar lazos de cooperación con el mundo escolar y desarrollar iniciativas de participación ciudadana, además de abrir un campo de fuentes inexplorado para la investigación.

El proyecto mencionado devino en la publicación de la Historia de la Educación en Chile (SERRANO SOL; RENGIFO, 2012), uno de los estudios más recientes sobre el tema, que aborda el asunto desde una perspectiva social y cultural concluyendo que si bien el sistema nacional de educación fue impulsado por el Estado, las comunidades locales fueron relevantes en relación a la oferta y demanda de educación. Las fuentes de los archivos liceanos jugaron un rol destacado en esa investigación ya que los trabajos en este ámbito se han construido mayormente en base a fuentes oficiales, es decir a las conservadas en el Archivo Nacional de Chile por mandato de la administración central. Los archivos escolares entregan nuevas pistas de indagación: quiénes fueron al liceo, de dónde venían, que profesiones tenían sus padres o 
apoderados, qué rol jugaban las comunidades locales en la oferta y demanda de educación, cuál era el comportamiento de autoridades y profesores, etc.

\section{METODOLOGÍA}

Actualmente, el programa enfoca su quehacer en cuatro ejes de gestión subdivididos en diversas líneas de acción: 1. Archivística y Patrimonio, 2. Innovación en docencia, 3. Investigación, 4. Impacto en la Comunidad. La metodología desarrollada es interdisciplinaria e intervienen profesionales de la archivística, la historia, la pedagogía, la didáctica, la conservación y las ciencias de la información para la elaboración de recursos digitales.

El eje de Archivística y Patrimonio apunta al conocimiento, habilitación y difusión de los archivos y colecciones conservadas por los liceos. En una etapa inicial, el programa y las autoridades escolares gestionan proyectos para la habilitación de espacios y condiciones adecuadas para la instalación y conservación de sus archivos y bibliotecas históricas con el fin de darles una existencia formal dentro del ámbito escolar. Habitualmente, el material se encuentra desorganizado y sin protección y no existen políticas patrimoniales ni incentivos para su resguardo a nivel nacional, menos aún apoyos institucionales para su conservación. Los primeros liceos en constituir formalmente sus archivos fueron el Abate Molina de Talca (1823), Neandro Schilling (1846) y Eduardo Charme (1905) de San Fernando. Luego vinieron el Instituto Nacional General José Miguel Carrera (1813), el Liceo de Niñas $N^{\circ} 1$ (1894) y el Liceo de Aplicación (1893), ubicados en la ciudad de Santiago. Por último, el Liceo Gregorio Cordovéz de La Serena (1821), el primero fundado en la zona norte de Chile, inauguró su archivo en junio de 2016.

En paralelo, el programa ha realizado el censo y toma de muestras digitales de los documentos de una veintena de liceos a lo largo del país que están en proceso de conformar sus archivos. La etapa descrita es relevante ya que establece una plataforma desde la cual es posible implementar los pasos siguientes de innovación pedagógica, investigación académica y difusión. Asimismo, en esta primera etapa se realizan las gestiones para la generación de vínculos institucionales entre liceos y universidad, esta última como institución gestora y patrocinadora. La relación que se establece es de primera importancia ya que los profesores del sistema 
secundario público chileno están habitualmente desvinculados del quehacer académico y universitario, lo que el programa busca revertir mediante instancias de trabajo colaborativo con investigadores y profesionales de diversas disciplinas y estudiantes de las academias y talleres que ellos dirigen. Proyectos enfocados en la recuperación y organización de los archivos, realización de inventarios, catálogos y manuales, postulación a concursos para implementar la infraestructura requerida, capacitación de personal encargado, talleres docentes, desarrollo de plataformas web son algunas de las posibilidades.

El propósito es continuar replicando este modelo a nivel nacional, elaborando un diagnóstico que permita implementar formas adecuadas y particulares de trabajo, investigación y gestión para cada liceo.

El eje de Innovación en docencia, y la profundización de elementos de formación ciudadana, es un objetivo crucial del proyecto y, probablemente, el más desafiante. Así también, construir un modelo didáctico para el sector curricular de Historia, Geografía y Ciencias Sociales en base al uso en aula de fuentes documentales. Se enfoca en el desarrollo de didácticas innovadoras en la formación de profesores y estudiantes secundarios y universitarios mediante el uso de archivos y colecciones patrimoniales y apunta, precisamente, al conocimiento y la valoración por parte de los jóvenes de las instituciones públicas y privadas que funcionan dentro de la sociedad. Los estudiantes secundarios, guiados por docentes e investigadores universitarios en conjunto con sus propios profesores, convierten los archivos de sus liceos en verdaderos laboratorios de aprendizaje, investigación y trabajo colaborativo. De esta forma, ellos son partícipes y gestores de la puesta en valor de su patrimonio histórico y cultural, que contribuye a generar vínculos de pertenencia con el medio local desde el reconocimiento de un pasado común. EL modo de trabajo consiste en elaborar criterios y propuestas a desarrollar una vez concluidas las labores de organización y habilitación del archivo, también realizadas en este contexto. Además de ello, y debido a la falta de recursos de los planteles públicos, se idean conjuntamente proyectos que concursan para obtener fondos que incrementan las posibilidades de acción del programa. Con apoyo de financiamiento estatal y privado se han realizado por ejemplo, talleres enfocados en la digitalización, descripción y conservación de la serie de Correspondencia Enviada por el rector del IN entre 1824 y 1900; la transcripción y digitalización de las fichas de matrícula de los estudiantes del Liceo Abate Molina; el estudio y la elaboración de fichas de 
investigación del Boletín del Instituto Nacional creado en 1936; el catastro de los objetos patrimoniales del Liceo de Aplicación; la organización de contenidos en base al archivo del Liceo $\mathrm{N}^{\circ} 1$ de Santiago para ser presentados en el Día del Patrimonio Nacional, entre otros. La hipótesis de este segundo eje, Innovación en docencia, es que la experiencia de trabajo fuera del espacio tradicional del aula, con un material desconocido y novedoso como son los documentos y fuentes primarias y proyectado a las necesidades de la sociedad contemporánea, mejora las habilidades de crítica e indagación histórica y el rendimiento en el aprendizaje de la historia y las ciencias sociales. A la vez, estas acciones construyen una paulatina revalorización del liceo como centro cívico, de desarrollo de la cultura local, en donde los propios estudiantes ven reflejados el pasado no sólo de su escuela y ciudad sino también de su entorno familiar. Este hecho otorga a la experiencia pedagógica un invalorable sentido de identidad y pertenencia.

El eje de Investigación se refiere al fomento de la investigación académica, como también a la experiencia del archivo y del patrimonio cultural de estudiantes universitarios dentro de su proceso formativo. Junto a esto, el incentivo al desarrollo de líneas de investigación interdisciplinarias tales como gestión de documentos y archivos, gestión del patrimonio histórico educativo, ciudadanía, transparencia y acceso a la información pública, entre otras. Sus objetivos nacieron ligados a los desafíos que impuso la investigación citada sobre la historia de la educación en Chile y la necesidad de contar con nuevas fuentes primarias para una mirada revisada y complejizada del proceso.

El hallazgo del archivo histórico del liceo Abate Molina ${ }^{6}$ y luego de los otros que fueron conformando la red, todos fundados mientras se construía la nueva nación, marca probablemente un punto de inflexión en la historiografía chilena porque devela un corpus de fuentes inexplorado que debe terminar de sistematizarse para habilitar su información. En general, la historia de la educación chilena ha sido construida en base a archivos oficiales emitidos por entidades administrativas como ministerios, intendencias o municipalidades. Los documentos guardados indistintamente por las autoridades de los liceos, y que habitualmente difieren de los que la administración estatal ordenó preservar en cada época, han sido escasamente consultados por

\footnotetext{
${ }^{6}$ La apertura del archivo del Liceo Abate Molina permitió el uso de nuevas metodologías cuantitativas a través del estudio de la serie de fichas de matrícula de los alumnos de las primeras décadas del siglo XX.
} 
historiadores o estudiosos de otras disciplinas. El Archivo Nacional de Chile no alberga copia de toda esta información cuyas actuales condiciones físicas y la carencia de una política nacional de archivos que apoye su sobrevivencia, ponen en riesgo su conservación. Es prioritario iniciar procesos de digitalización de su contenido y difusión a una comunidad académica, educacional y ciudadana más amplia.

El eje de Impacto en la comunidad persigue el fortalecimiento de procesos de memoria, apropiación y valorización del patrimonio escolar mediante la consolidación de una red nacional de archivos escolares patrocinada por la Pontificia Universidad Católica de Chile. Junto a esto, se busca promover la gestión de las propias instituciones escolares para la conservación y difusión de su patrimonio dado el aporte que esto significa para el desarrollo de la cultura local.

La difusión de las acciones que promueve el programa es fundamental en el proceso de conformación de una ciudadanía escolar informada y participativa. Desde 2015, se ha creado una instancia para la presentación abierta de resultados de los proyectos realizados por los equipos escolares y universitarios ante un público amplio y multidisciplinario. Se trata del Congreso Nacional de Educación y Patrimonio inaugurado en el Liceo Gregorio Cordovéz de la ciudad de La Serena en 2015, y que se llevará a cabo en el Instituto Nacional General José Miguel Carrera en octubre de 2016. Este evento, que se convoca a todos los actores comprometidos con la educación acoge a los proyectos adjuntos al programa que se encuentran en diferentes estados de avance.

La paulatina creación del Archivo Digital de la Educación es la instancia enfocada a la participación y uso de los fondos por parte de la comunidad global para, entre otras cosas, incentivar el desarrollo de un material adecuado para implementar proyectos en humanidades digitales. Es, además, una forma de "llegar a públicos diversos y alentar la participación popular en la presentación y preservación del pasado" (LEON, 2010). La consolidación de una plataforma digital que albergue los productos y resultados de los trabajos ya elaborados y en desarrollo es una de las prioridades a corto plazo. El programa ha identificado la necesidad de establecer diferentes herramientas digitales para responder a las características de las fuentes y a los diversos públicos que eventualmente están interesados en el acceso a estas. Estas definiciones no han sido parte de un estudio de usuarios sino que se han establecido manera apriori. En ese 
sentido, y a modo de ejemplo, presentamos cuatro productos de acceso público que dan cuenta de estas definiciones y orientaciones:

1) Directorio de Matrícula del Liceo Abate Molina de Talca. Desarrollado como tabla de datos el directorio permite acceder a los registros y digitalizaciones de matrícula de alumnos del Liceo Abate Molina de Talca entre los años 1854 y 1930. Está enfocado en poner a disposición del público general y de investigadores un recurso informativo que diera cuenta de la rica información nominal -de alumnos, padres, apoderados- que tanto permita una búsqueda particular como acceder a la totalidad de los registros digitalizados. El involucramiento de los estudiantes del liceo en el desarrollo de esta plataforma también los constituye en público objetivo de esta herramienta.

2) Correspondencia enviada por el Rector del Instituto Nacional General José Miguel Carrera. Desarrollado como catálogo de serie, está descrito bajo normas internacionales de descripción archivística y soportado en el programa Acces To Memory -ATOM- desarrollado por Artefactual Systems, enfocándose en la comunidad de investigadores interesados en los diversas series documentales que posee el archivo del liceo. El involucramiento de los estudiantes del liceo en el desarrollo de esta plataforma también los constituye en público objetivo de esta herramienta.

3) Colecciones Digitales. Esta es una muestra de los documentos que hemos catastrado en los liceos a través de estos seis años de trabajo. La idea es disponer de un corpus muy acotado de fuentes digitales seleccionadas por liceo que den cuenta del valor informativo de los documentos que resguardan las instituciones educativas y que puedan ser utilizadas por los profesores en el desarrollo de actividades dentro y fuera del aula. Cumple una función divulgativa general y se ha implementado en el programa para colecciones digitales Omeka desarrollado por $\underline{\text { Roy }}$ $\underline{\text { Rosenzweig Center for History and New Media, de la George Mason University. }}$

4) Catálogo de la Biblioteca Histórica del Instituto Nacional General José Miguel Carrera. Elaborado en la necesidad de controlar la colección histórica de la biblioteca de dicha institución y que posibilita, en particular a los investigadores, acceder a una amplia bibliografía utilizada en el establecimiento durante el siglo XIX y principios del siglo XX. Da cuenta además del proceso 
interno de crecimiento y pérdida bibliográfica sufrida por la institución. En este proyecto se desarrolló utilizando el programa open source OpenBiblio.

Estas primeras iniciativas en el ámbito digital son exploraciones que de ninguna manera se proponen como definitivas, forman parte del repertorio amplio que posibilitan los archivos en el mundo digital y tratan de incentivar el desarrollo de la historia digital. Todas estas plataformas se encuentran disponibles a través de enlaces en <www.archivosescolares.cl>.

El proyecto contempla además el registro audiovisual de todas las acciones desarrolladas en los diversos liceos. El guion general se sustenta en las necesidades comunicativas hacia los actores involucrados como en la difusión de la labor realizada, y forma parte de una estrategia que permite sostener un relato dinámico e integrador.

\section{LOGROS Y RESULTADOS}

En su fase inicial el Programa de Archivos Escolares ha realizado una completa intervención enfocada en la recuperación, censo y organización de los archivos escolares adjuntos, además de la realización del censo de cada uno de los establecimientos secundarios regionales que incluye el proyecto. En este sentido, se cumplió con el objetivo de hallar fuentes primarias que rebasaran el material oficial conservado en el Archivo Nacional de Chile. En paralelo, se inició un proceso de vinculación y asociación entre el programa y los liceos seleccionados cumpliéndose así un segundo objetivo prioritario de generar vínculos con las comunidades escolares para el desarrollo de competencias en el sector de Historia, Geografía y Ciencias Sociales, así como con el fortalecimiento de la identidad docente. El énfasis hacia la construcción de lazos con la comunidad deriva, en parte, del diagnóstico que emitió la Comisión de Formación Ciudadana, convocada en 2004 por el Ministerio de Educación de Chile, en el cual se relevó la necesidad de dotar a los profesores de una nueva mirada a su propio quehacer y de la importancia que este ha tenido en el proceso de modernización de la sociedad chilena.

En la experiencia han participado, además de la comunidad escolar -directivos, docentes, estudiantes-, historiadores y archiveros. En pos de la recuperación del patrimonio documental e iniciando, mediante la realización de talleres y actividades, procesos de 
valorización patrimonial dentro de la comunidad, se incentiva la relación del archivo con el aula como con la identidad docente y escolar. Asimismo, se ha logrado demostrar la necesidad de que cada institución tome en sus manos la gestión y comunicación de su patrimonio documental y desarrollen desde ellas reflexiones vinculadas a políticas de archivo. En este sentido, se ha detectado la necesidad de generar mecanismos que estrechen la relación entre patrimonio y sociedad, ya que existe una cierta desvinculación entre lo conservado y quienes conservan.

El modelo de intervención puede ser problematizado a la vez que presenta ciertas claves que constituyen elementos básicos para la discusión de una nueva ley de archivos en Chile. A partir de este caso particular se proyectan diversos niveles de análisis de la relación del archivo con los investigadores y la docencia escolar y universitaria, permitiendo por abordar la situación de los archivos en las instituciones públicas desde variables como el acceso, la descentralización del patrimonio de los archivos, la enseñanza de la historia, el papel de los archiveros, entre otras.

\section{BIBLIOGRAFÍA}

ALBERCH, Ramón y BOADAS, Joan. La función cultural de los archivos. Ikerlanak III, Bergara, Eusko Jaurlaritzaren Argitalpen Zerbitzu-Nagusia, Servicio Central de Publicaciones del Gobierno Vasco, 1991. Disponible en: 〈http://www.snae.org/pdf/ikerlanak3.pdf>.

AMUNÁTEGUI, S., Domingo. Los primeros años del Instituto Nacional (1813-1835). Santiago: Imprenta Cervantes, 1889.

CRUZ, Nicolás. El Surgimiento de la Educación Secundaria Pública en Chile. 1843 - 1876. (El plan de Estudios Humanista). Santiago: Ediciones de la Dirección de Bibliotecas, Archivos y Museos, 2002.

CRUZ, José Ramón (ed.). Administración de documentos y archivos. Textos fundamentales. Madrid: Coordinadora de Asociaciones de Archiveros, Ministerio de Cultura, 2011. Disponible en: <http://www.archiveros.net/LIBRO.ARCHIVOS.IBEROAMERICANOS.pdf>.

DICCIONARIO de Terminología Archivística. Ministerio de Cultura de España, 1995. Disponible en: <http://www.mecd.gob.es/cultura-mecd/areascultura/archivos/mc/dta/diccionario.html >.

GUÍAS de Capacitación. Dirección de Bibliotecas, Archivos y Museos, DIBAM. Programa Memorias Siglo XX. Disponible en: <www.memoriadelsigloxx.cl>. 
HEREDIA H., Antonia. ¿Qué es un archivo? España: Ediciones Trea S.L., 2007.

LABARCA, Amanda. Historia de la enseñanza en Chile. Santiago: Imprenta Universitaria, 1939.

LEON, Sharon. Doing History in Public: Digital History in the Digital Humanities. Maryland: Institute for Technology in the Humanities, 2010.

LOS ARCHIVOS de las Ong. Una Memoria Para Compartir. Guía Práctica en 60 Preguntas.

Consejo Internacional de Archivos. Disponible

en: <http://www.arxivers.org/docs/archivos\%20y\%20ONGs.pdf>.

LUZ, Joel Martins. O Arquivo escolar para la historia das instiuicoes educacionais. Maestrando do PPGEdu/ UFMT/CUR, 2014.

MENEZES Maria Cristina; SILVA, Cristina Leite da; TEIXEIRA JÚNIOR, Oscar. O arquivo escolar: lugar da memória, lugar da história. Horizontes, v. 23, n. 1, p. 67-76, jan./jun. 2005.

MULTILINGUAL Archive Terminology. Consejo Internacional de Archivos, ICA. Disponible en: <http://www.ciscra.org/mat>.

SERRANO SOL, Macarena Ponce de León y RENGIFO, Francisca. Historia de la educación en Chile (1810-2010). Santiago: Editorial Taurus, 2012.

SERRANO SOL. Universidad y Nación. Chile en el siglo XIX. Santiago: Editorial Universitaria, 1994. 